\title{
Dynamics on resonant clusters for the quintic non linear Schrödinger equation
}

\author{
Emanuele Haus and Laurent Thomann \\ Communicated by Y. Charles Li, received October 26, 2012.
}

\begin{abstract}
We construct solutions to the quintic nonlinear Schrödinger equation on the circle

$i \partial_{t} u+\partial_{x}^{2} u=\nu|u|^{4} u, \quad \nu \ll 1, x \in \mathbb{S}^{1}, t \in \mathbb{R}$,

with initial conditions supported on arbitrarily many different resonant clusters. This is a sequel the work [5] of Benoît Grébert and the second author.
\end{abstract}

\section{Contents}

\begin{tabular}{|ll|}
\hline $1 . \quad$ Introduction and results & 157 \\
\hline 2. Existence of resonant sets & 160 \\
\hline 3. The model equation & 163 \\
\hline 4. The perturbation analvsis and proof of the main results & 167 \\
\hline References & 169 \\
\hline
\end{tabular}

\section{Introduction and results}

1.1. General introduction. In this paper we consider the quintic nonlinear periodic Schrödinger equation

$$
\left\{\begin{array}{l}
i \partial_{t} u+\partial_{x}^{2} u=\nu|u|^{4} u, \quad(t, x) \in \mathbb{R} \times \mathbb{S}^{1}, \\
u(0, x)=u_{0}(x),
\end{array}\right.
$$

where $\nu>0$ is some small parameter. In [5] B. Grébert and the second author showed a beating effect for (1.1): there exist solutions which are supported on 4 so-called resonant modes and which stay close to a time-periodic solution for long time. These solutions moreover show that there is an energy exchange between the

1991 Mathematics Subject Classification. 37K45, 35Q55, 35B34, 35B35.

Key words and phrases. Nonlinear Schrödinger equation, Resonant normal form, energy exchange.

Both authors were supported in part by the grant ANR-10-JCJC 0109.

(C)2013 International Press 
considered modes, which is a genuine nonlinear effect. We call this mechanism a beating effect. Such a phenomenon was first observed by Grébert and Villegas-Blas 6] on a cubic Schrödinger equation. Let us also mention the work [4 by GrébertPaturel-Thomann where a general principle is extracted so that such a phenomenon occurs. Finally we refer to the introduction of $\mathbf{5}$ for more results on the long time dynamics for 1.10 and related models.

Our aim here is to extend the main result of $[5$ and show that we can construct solutions to (1.1) supported on arbitrarily many resonant modes.

Recall [5. Definition 1.1] that a set of the form

$$
\{n, n+3 m, n+4 m, n+m\}, \quad m \in \mathbb{Z} \backslash\{0\} \text { and } n \in \mathbb{Z},
$$

is called a resonant set. These sets exactly correspond to the resonant monomials of order 6 of the Hamiltonian of (1.1) which contain 4 different modes. Here, we consider resonant sets of the type

$$
\mathcal{A}_{k}=\left\{n_{k}-2, n_{k}-1, n_{k}+1, n_{k}+2\right\},
$$

where $\left(n_{k}\right)_{k \geq 1}$ is a sequence of integers which will be described. In the sequel, we denote by

$$
a_{2}^{(k)}=n_{k}-2, \quad a_{1}^{(k)}=n_{k}+1, \quad b_{2}^{(k)}=n_{k}+2, \quad b_{1}^{(k)}=n_{k}-1,
$$

and for $K \geq 1$, we define

$$
\mathcal{A}=\bigcup_{k=1}^{K} \mathcal{A}_{k} .
$$

To begin with, let us recall the result of [5] for the resonant set $\mathcal{A}_{k}$.

TheOREm 1.1. [5] Let $k \geq 1$. There exist $T_{k}>0, \nu_{0}>0, \alpha_{k} \in(0,1 / 2)$ and a $2 T_{k}$-periodic function $K_{\star}^{(k)}: \mathbb{R} \longmapsto(0,1)$ which satisfies $K_{\star}^{(k)}(0) \leq \alpha_{k}$ and $K_{\star}^{(k)}\left(T_{k}\right) \geq 1-\alpha_{k}$ such that if $0<\nu<\nu_{0}$, there exists a solution $u_{k}$ to (1.1) satisfying for all $|t| \leq \nu^{-9 / 8}$

$$
u_{k}(t, x)=v_{k}(t, x)+\nu^{1 / 4} q(t, x),
$$

with

$$
v_{k}(t, x)=\sum_{j \in \mathcal{A}_{k}} w_{j}(t) \mathrm{e}^{i j x},
$$

and

$$
\begin{aligned}
\left|w_{a_{1}^{(k)}}(t)\right|^{2} & =2\left|w_{a_{2}^{(k)}}(t)\right|^{2}=K_{\star}^{(k)}(\nu t) \\
\left|w_{b_{1}^{(k)}}(t)\right|^{2} & =2\left|w_{b_{2}^{(k)}}(t)\right|^{2}=1-K_{\star}^{(k)}(\nu t),
\end{aligned}
$$

and $q$ is smooth in time and analytic in space on $\left[-\nu^{-9 / 8}, \nu^{-9 / 8}\right] \times \mathbb{S}^{1}$. Moreover, the Fourier coefficients $\widehat{q}_{j}(t)$ of $q(t)$ satisfy

$$
\sup _{|t| \leq \nu^{-9 / 8}}\left|\widehat{q}_{j}(t)\right| \leq C \mathrm{e}^{-|j|}
$$

with $C$ independent of $k \geq 1$ and $\nu>0$.

The result is not exactly stated like this in [5], but is proven there. In particular, the analyticity of the remainder term follows from the analytical framework of the Birkhoff normal form procedure in [5] Section 3]. See also 4 .

Theorem 1.1 shows that there are non trivial interactions between the modes in $\mathcal{A}_{k}$ and they occur for times $t \sim \nu^{-1} T_{k}$. 
1.2. The main result. In this paper we prove that if the resonant sets are carefully chosen, there exist solutions to (1.1) which are the superposition of solutions of the previous type.

THEOREM 1.2. There exists $\nu_{0}>0$ and there exists an increasing sequence of integers $\left(n_{k}\right)_{k \geq 1}$ such that, if $0<\nu<\nu_{0}$, for all $K \geq 1$ with $n_{K} \leq-(1 / 32) \ln \nu$ there exists a solution to (1.1) which reads for all $|t| \leq \nu^{-9 / 8}$

$$
u(t, x)=\sum_{k=1}^{K} \mathrm{e}^{-n_{k}} v_{k}(t, x)+\nu^{1 / 4} q(t, x)
$$

where

i) For all $1 \leq k \leq K, v_{k}$ is as in Theorem 1.1.

ii) The error term is smooth in time and analytic in space on $\left[-\nu^{-9 / 8}, \nu^{-9 / 8}\right] \times \mathbb{S}^{1}$. Moreover, the Fourier coefficients $\widehat{q}_{j}(t)$ of $q(t)$ satisfy

$$
\sup _{|t| \leq \nu^{-9 / 8}}\left|\widehat{q}_{j}(t)\right| \leq C \mathrm{e}^{-|j|}
$$

with $C$ independent of $K \geq 1$ and $\nu>0$.

This result shows a beating effect inside of each resonant set, but there is no energy transfer between two different resonant clusters. In particular, we do not show an energy transfer from the low to the high frequencies.

In our example, for all $s \geq 0,\|u\|_{H^{s}}$ is almost preserved during the time. This is due to the particular form (1.3) of our resonant sets. We believe that a similar construction for more general resonant sets (1.2) can be done.

For all $j \in \mathbb{Z}$, the Fourier coefficient $\widehat{u}_{j}$ of $u$ in 1.5 satisfies

$$
\sup _{|t| \leq \nu^{-9 / 8}}\left|\widehat{u}_{j}(t)\right| \leq C \mathrm{e}^{-|j|}
$$

thus $u$ is bounded in an analytic norm uniformly in $K \geq 1$ for this time scale. A natural question is whether we can choose $K=+\infty$ in Theorem 1.2 Our method does not allow this extension since the period of $v_{k}$ grows to infinity with $k$. Moreover, the expansion in (1.5) is relevant as long as $\mathrm{e}^{-n_{k}} v_{k}$ is larger than the error term, and this gives the limitation $n_{K} \leq-c \ln \nu$.

In fact there are many sequences which satisfy Theorem 1.2 almost all sequences which satisfy $n_{k+1} \geq 12 n_{k}^{2}$ can be taken (see the proof of Proposition 2.4).

Our approach allows also to treat the focusing Schrödinger equation $(\nu<0)$, but for simplicity we only deal with the case $\nu>0$.

With an appropriate choice of the initial conditions, we can construct quasiperiodic solutions in time for a large set of frequencies.

Corollary 1.3. For every $K \geq 1$ and every sequence of real numbers

$$
\Lambda_{1}, \Lambda_{2}, \ldots, \Lambda_{K}>0
$$

, there exists $N \in \mathbb{N}$ so that, if $\nu>0$ is small enough, we can construct $v_{k}$ of period $2 N \Lambda_{k} / \nu$ in time.

This is clearly a nonlinear phenomenon, since in the linear regime all the frequencies are integer multiples of the same number. 
We remark that Theorem 1.2 is not a direct consequence of Theorem 1.1 Indeed, when considering multiple clusters, new interactions could a priori appear among the clusters. Moreover, since with several clusters the structure of the frequency set is more complicated, it also becomes more difficult to keep under control the interaction of the multiple-cluster set with the rest of the Fourier modes. Both these points require a careful choice of the sequence $\left(n_{k}\right)$, which involves both separation of the clusters and arithmetical properties of the $n_{k}$ 's.

1.3. Plan of the paper. In Section 2 we prove the existence of resonant sets made up of several clusters which do not interact much with one another. In Section 3 we recall the Hamiltonian structure of (1.1) and we study the model equation, which is obtained by truncating the error terms of the normal form (higher order terms and terms involving frequencies outside the resonant sets). In Section 4 we perform the perturbation analysis and collect the results of the previous sections in order to prove our main results. Throughout the paper, we widely rely on the results obtained by B. Grébert and the second author in 5]: since several proofs turn out to be similar, here we choose to highlight what is new and different, rather than copy out the proofs in $[\mathbf{5}]$.

\section{Existence of resonant sets}

In this section, we show the existence of a sequence $\left(n_{k}\right)$ such that the modes in $\mathcal{A}_{k}$ and $\mathcal{A}_{j}$ do not interact much when $k<j$. We will see that this is ensured when $\left(n_{k}\right)$ is growing fast enough and if it satisfies some arithmetical condition.

Define the set

$$
\begin{aligned}
\mathcal{R}:= & \left\{\left(j_{1}, j_{2}, j_{3}, \ell_{1}, \ell_{2}, \ell_{3}\right) \in \mathbb{Z}^{6} \text { s.t. } \quad j_{1}+j_{2}+j_{3}=\ell_{1}+\ell_{2}+\ell_{3}\right. \\
& \text { and } \left.j_{1}^{2}+j_{2}^{2}+j_{3}^{2}=\ell_{1}^{2}+\ell_{2}^{2}+\ell_{3}^{2}\right\} .
\end{aligned}
$$

We first recall a couple of results from [5] (namely, [5, Lemma 2.1] and [5. Lemma 2.4]).

Lemma 2.1. Assume that $\left(j_{1}, j_{2}, j_{3}, \ell_{1}, \ell_{2}, \ell_{3}\right) \in \mathcal{R}$ and $\left\{j_{1}, j_{2}, j_{3}\right\} \neq\left\{\ell_{1}, \ell_{2}, \ell_{3}\right\}$. Then $\left\{j_{1}, j_{2}, j_{3}\right\} \cap\left\{\ell_{1}, \ell_{2}, \ell_{3}\right\}=\emptyset$.

Lemma 2.2. Fix $k \in \mathbb{Z}$. Let $\left(j_{1}, j_{2}, j_{3}, \ell_{1}, p_{1}, p_{2}\right) \in \mathcal{R}$. Assume that $j_{1}, j_{2}, j_{3}, \ell_{1} \in$ $\mathcal{A}_{k}$. Then $p_{1}, p_{2} \in \mathcal{A}_{k}$.

In the following lemma we prove that, if $\left(n_{k}\right)$ is growing fast enough, then there is no direct interaction among the clusters.

LEMMA 2.3. Assume that the sequence $\left(n_{k}\right)_{k \geq 1}$ satisfies $n_{1} \geq 3$ and $n_{k+1} \geq$ $12 n_{k}^{2}$. Then the following holds true: Let $\mathcal{S}:=\left\{j_{1}, j_{2}, j_{3}, \ell_{1}, \ell_{2}, \ell_{3}\right\} \subset \mathcal{A}$ with

$$
\left\{\begin{array}{l}
j_{1}^{2}+j_{2}^{2}+j_{3}^{2}=\ell_{1}^{2}+\ell_{2}^{2}+\ell_{3}^{2}, \\
j_{1}+j_{2}+j_{3}=\ell_{1}+\ell_{2}+\ell_{3},
\end{array} \quad \text { and } \quad\left\{j_{1}, j_{2}, j_{3}\right\} \neq\left\{\ell_{1}, \ell_{2}, \ell_{3}\right\} .\right.
$$

Then there exists $1 \leq k \leq K$ so that $\mathcal{S} \subset \mathcal{A}_{k}$.

Proof. Consider $\mathcal{S}=\left\{j_{1}, j_{2}, j_{3}, \ell_{1}, \ell_{2}, \ell_{3}\right\} \subset \mathcal{A}$ such that 2.1 holds. Assume that $\ell_{1}=\max \mathcal{S}$. Then $\ell_{1}=n_{k}+r_{1}$ for some $1 \leq k \leq K$ and $r_{1} \in\{-2,-1,1,2\}$. If $k=1$ we are done, hence we assume that $k \geq 2$. We claim that one of the integers 
$j_{1}, j_{2}, j_{3}$, say $j_{1}$, is of the form $j_{1}=n_{k}+q_{1}$ with $q_{1} \in\{-2,-1,1,2\}$. If it is not the case, $j_{1}, j_{2}, j_{3} \leq n_{k-1}+2$ and thus

$$
j_{1}^{2}+j_{2}^{2}+j_{2}^{2} \leq 3\left(n_{k-1}+2\right)^{2}<12 n_{k-1}^{2} \leq n_{k} \leq\left(n_{k}-2\right)^{2} \leq \ell_{1}^{2},
$$

which is a contradiction. We plug the expressions of $\ell_{1}$ and $j_{1}$ in (2.1) and obtain

$$
2\left(q_{1}-r_{1}\right) n_{k}=\ell_{2}^{2}+\ell_{3}^{2}-j_{2}^{2}-j_{3}^{2}+r_{1}^{2}-q_{1}^{2} .
$$

From Lemma 2.1] we have $q_{1} \neq r_{1}$.

- Assume that $\left\{\ell_{2}, \ell_{3}, j_{2}, j_{3}\right\} \subset \bigcup_{m=1}^{k-1} \mathcal{A}_{m}$. Then

$$
\begin{aligned}
\left|\ell_{2}^{2}+\ell_{3}^{2}-j_{2}^{2}-j_{3}^{2}+r_{1}^{2}-q_{1}^{2}\right| & \leq 6\left(n_{k-1}+2\right)^{2} \\
& <24 n_{k-1}^{2} \\
& \leq 2 n_{k} \\
& \leq 2\left|q_{1}-r_{1}\right| n_{k},
\end{aligned}
$$

which is a contradiction.

- We can therefore assume that $\left\{\ell_{2}, j_{2}\right\} \cap \mathcal{A}_{k} \neq \emptyset$. We consider the case $\ell_{2} \in \mathcal{A}_{k}$ (the proof is analogous in the case $j_{2} \in \mathcal{A}_{k}$ ). If $\left\{j_{2}, j_{3}\right\} \subset \bigcup_{m=1}^{k-1} \mathcal{A}_{m}$ we can show that

$$
2\left(q_{1}-r_{1}\right) n_{k}<\ell_{2}^{2}+\ell_{3}^{2}-j_{2}^{2}-j_{3}^{2}+r_{1}^{2}-q_{1}^{2},
$$

thus we can assume that $j_{2} \in \mathcal{A}_{k}$ and we write $j_{2}=n_{k}+q_{2}, \ell_{2}=n_{k}+r_{2}$. The relation (2.2) then reads

$$
2\left(q_{1}+q_{2}-r_{1}-r_{2}\right) n_{k}=\ell_{3}^{2}-j_{3}^{2}+r_{1}^{2}+r_{2}^{2}-q_{1}^{2}-q_{2}^{2} .
$$

With a similar argument we deduce that $\left\{\ell_{3}, j_{3}\right\} \subset \mathcal{A}_{k}$, which completes the proof.

Then we prove the analogous of Lemma 2.2 in the case of multiple clusters.

Proposition 2.4. There exists a sequence $\left(n_{k}\right)_{k \geq 1}$ which satisfies $n_{1} \geq 3$, $n_{k+1} \geq 12 n_{k}^{2}$ and so that the following non-resonance condition holds true: Let $\left(j_{1}, j_{2}, j_{3}, \ell_{1}, p_{1}, p_{2}\right) \in \mathcal{R}$. Assume that $j_{1}, j_{2}, j_{3}, \ell_{1} \in \mathcal{A}$. Then $p_{1}, p_{2} \in \mathcal{A}$.

Proof. We construct such a sequence $\left(n_{k}\right)_{k \geq 1}$ by induction. The case $K=1$ is given in Lemma 2.2 Now, assume that we have constructed the first $K$ elements of the sequence $\left(n_{k}\right)_{k=1}^{K}$. We will prove that we can choose $n_{K+1}$ satisfying the wanted properties.

Suppose that we have fixed $n_{K+1}$ (and therefore $\mathcal{A}_{K+1}$ ): we now investigate which arithmetical properties are required in order to satisfy the non-resonance condition defined in Proposition 2.4

Let $j_{1}, j_{2}, j_{3}, \ell_{1} \in \mathcal{A}$ and $p_{1}, p_{2} \in \mathbb{N}$ so that

$$
\left\{\begin{array}{l}
p_{1}+p_{2}=j_{1}+j_{2}+j_{3}-\ell_{1}=: S, \\
p_{1}^{2}+p_{2}^{2}=j_{1}^{2}+j_{2}^{2}+j_{3}^{2}-\ell_{1}^{2}=: T .
\end{array}\right.
$$

The two complex (possibly coinciding) solutions $\left(p_{1}, p_{2}\right)$ to (2.3) are the roots of the polynomial

$$
X^{2}-S X+\frac{1}{2}\left(S^{2}-T\right) .
$$

The discriminant of this polynomial is $\Delta=2 T-S^{2}$. Therefore, a necessary condition for (2.31) to have integer solutions is that $2 T-S^{2}$ is a perfect square. 
Each of the elements $j_{1}, j_{2}, j_{3}, \ell_{1}$ may belong either to $\mathcal{A}_{K+1}$ or to $\mathcal{A}_{k}$ with $k \leq K$. We have to distinguish 8 different cases, depending on how many of the $j$ 's belong to $\mathcal{A}_{K+1}$ (4 possibilities, from 0 to 3 ) and whether $\ell_{1} \in \mathcal{A}_{K+1}$ or not.

- Case $(0,0): j_{1}, j_{2}, j_{3}, \ell_{1} \notin \mathcal{A}_{K+1}$.

No further property has to be verified: the non-resonance condition is satisfied by induction hypothesis.

- Case $(1,0): j_{1} \in \mathcal{A}_{K+1}, j_{2}, j_{3}, \ell_{1} \notin \mathcal{A}_{K+1}$.

We exploit the identity

$\Delta=2\left(j_{1}^{2}+j_{2}^{2}+j_{3}^{2}-\ell_{1}^{2}\right)-\left(j_{1}+j_{2}+j_{3}-\ell_{1}\right)^{2}=\left(j_{1}-j_{2}-j_{3}+\ell_{1}\right)^{2}-4\left(\ell_{1}-j_{2}\right)\left(\ell_{1}-j_{3}\right)$.

Now, $j_{1}$ may be expressed as $j_{1}=n_{K+1}+c_{1}$, with $c_{1} \in\{-2,-1,1,2\}$. Therefore

$$
\Delta=\left(n_{K+1}+c_{1}-j_{2}-j_{3}+\ell_{1}\right)^{2}-4\left(\ell_{1}-j_{2}\right)\left(\ell_{1}-j_{3}\right) .
$$

The relevant thing here is that $\Delta$ has the form $\left(n_{K+1}+\tilde{c}_{1}\right)^{2}+\tilde{c}_{2}$. If $\tilde{c}_{2}=0$, then either $\ell_{1}=j_{2}$ or $\ell_{1}=j_{3}$, which implies the non-resonance condition $p_{1}, p_{2} \in \mathcal{A}$, by Lemma 2.1] If $\tilde{c}_{2} \neq 0$, it is sufficient to choose $n_{K+1}$ large enough to prevent $\Delta$ from being a perfect square.

- Case $(2,0): j_{1}, j_{2} \in \mathcal{A}_{K+1}, j_{3}, \ell_{1} \notin \mathcal{A}_{K+1}$.

We have $j_{1}=n_{K+1}+c_{1}, j_{2}=n_{K+1}+c_{2}$ with $c_{1}, c_{2} \in\{-2,-1,1,2\}$. Thus,

$$
\begin{aligned}
\Delta= & 2\left[\left(n_{K+1}+c_{1}\right)^{2}+\left(n_{K+1}+c_{2}\right)^{2}+j_{3}^{2}-\ell_{1}^{2}\right] \\
& -\left[\left(n_{K+1}+c_{1}\right)+\left(n_{K+1}+c_{2}\right)+j_{3}-\ell_{1}\right]^{2}= \\
= & -4\left(j_{3}-\ell_{1}\right) n_{K+1}+\left(c_{1}-c_{2}\right)^{2}-2\left(c_{1}+c_{2}\right)\left(j_{3}-\ell_{1}\right)+\left(j_{3}-\ell_{1}\right)\left(j_{3}+3 \ell_{1}\right) .
\end{aligned}
$$

If $\ell_{1}=j_{3}$, then $p_{1}, p_{2} \in \mathcal{A}$, by Lemma 2.1] If $\ell_{1} \neq j_{3}$, then $\Delta$ has the form $\alpha n_{K+1}+\beta$, with $\alpha \neq 0$.

- Case $(3,0): j_{1}, j_{2}, j_{3} \in \mathcal{A}_{K+1}, \ell_{1} \notin \mathcal{A}_{K+1}$.

The conditions $n_{1} \geq 3, n_{k+1} \geq 12 n_{k}^{2}$ imply that if $k_{1} \neq k_{2}$ then $\left|n_{k_{1}}-n_{k_{2}}\right| \geq 105=$ $12 \cdot 3^{2}-3$. Therefore we exploit the translation invariance of the resonant set $\mathcal{R}$ and we translate back $\left(j_{1}, j_{2}, j_{3}, \ell_{1}, p_{1}, p_{2}\right)$ obtaining the new resonant sextuple

$$
\begin{aligned}
& \left(\tilde{j}_{1}, \tilde{j}_{2}, \tilde{j}_{3}, \tilde{\ell}_{1}, \tilde{p}_{1}, \tilde{p}_{2}\right):= \\
& \left(j_{1}-n_{K+1}, j_{2}-n_{K+1}, j_{3}-n_{K+1}, \ell_{1}-n_{K+1}, p_{1}-n_{K+1}, p_{2}-n_{K+1}\right)
\end{aligned}
$$

with $\left|\tilde{j}_{1}\right|,\left|\tilde{j}_{2}\right|,\left|\tilde{j}_{3}\right| \leq 2$ and $\left|\tilde{\ell}_{1}\right| \geq 105-2=103$, which is clearly absurd since

$$
\tilde{j}_{1}^{2}+\tilde{j}_{2}^{2}+\tilde{j}_{3}^{2}=\tilde{\ell}_{1}^{2}+\tilde{p}_{1}^{2}+\tilde{p}_{2}^{2} .
$$

- Case $(0,1): \ell_{1} \in \mathcal{A}_{K+1}, j_{1}, j_{2}, j_{3} \notin \mathcal{A}_{K+1}$.

This case is easily seen to be absurd, since

$$
j_{1}^{2}+j_{2}^{2}+j_{3}^{2}=\ell_{1}^{2}+p_{1}^{2}+p_{2}^{2}
$$

and $\ell_{1}$ is much bigger than $j_{1}, j_{2}, j_{3}$.

- Case $(1,1): j_{1}, \ell_{1} \in \mathcal{A}_{K+1}, j_{2}, j_{3} \notin \mathcal{A}_{K+1}$.

We write $j_{1}=n_{K+1}+c_{1}, \ell_{1}=n_{K+1}+c_{2}$ with $c_{1}, c_{2} \in\{-2,-1,1,2\}$. So we have

$$
\begin{aligned}
\Delta= & 2\left[\left(n_{K+1}+c_{1}\right)^{2}+j_{2}^{2}+j_{3}^{2}-\left(n_{K+1}+c_{2}\right)^{2}\right] \\
& -\left[\left(n_{K+1}+c_{1}\right)+j_{2}+j_{3}-\left(n_{K+1}+c_{2}\right)\right]^{2}= \\
= & 4\left(c_{1}-c_{2}\right) n_{K+1}+2\left(c_{1}^{2}+j_{2}^{2}+j_{3}^{2}-c_{2}^{2}\right)-\left(c_{1}+j_{2}+j_{3}-c_{2}\right)^{2} .
\end{aligned}
$$

If $c_{1}=c_{2}$, then $j_{1}=\ell_{1}$ and $p_{1}, p_{2} \in \mathcal{A}$ because of Lemma 2.1 Otherwise, $\Delta$ has the form $\alpha n_{K+1}+\beta$, with $\alpha \neq 0$. 
- Case $(2,1): j_{1}, j_{2}, \ell_{1} \in \mathcal{A}_{K+1}, j_{3} \notin \mathcal{A}_{K+1}$.

We have $j_{1}=n_{K+1}+c_{1}, j_{2}=n_{K+1}+c_{2}, \ell_{1}=n_{K+1}+c_{3}$ with $c_{1}, c_{2}, c_{3} \in$ $\{-2,-1,1,2\}$. Therefore

$$
\begin{aligned}
\Delta= & 2\left[\left(n_{K+1}+c_{1}\right)^{2}+\left(n_{K+1}+c_{2}\right)^{2}+j_{3}^{2}-\left(n_{K+1}+c_{3}\right)^{2}\right] \\
& -\left(n_{K+1}+c_{1}+c_{2}+j_{3}-c_{3}\right)^{2}= \\
= & \left(n_{K+1}+c_{1}+c_{2}-c_{3}-j_{3}\right)^{2}-4\left(c_{3}-c_{1}\right)\left(c_{3}-c_{2}\right),
\end{aligned}
$$

which has the same structure as for the case $(1,0)$ and therefore $\Delta$ is not a perfect square provided that $n_{K+1}$ is large enough.

- Case $(3,1): j_{1}, j_{2}, j_{3}, \ell_{1} \in \mathcal{A}_{K+1}$.

Then we have directly $p_{1}, p_{2} \in \mathcal{A}_{K+1} \subset \mathcal{A}$, by Lemma 2.2

Now, what still has to be proved is that we can choose $n_{K+1}$ arbitrarily large and such that $\alpha_{r} n_{K+1}+\beta_{r}$ is not a square for a finite number of couples of integers $\left\{\left(\alpha_{r}, \beta_{r}\right)\right\}_{r=1}^{s}$. We can limit ourselves to $\alpha_{r}>0$, since the conditions to be satisfied yield $\alpha_{r} \neq 0$ and, if $\alpha_{r}<0$, then $\alpha_{r} n_{K+1}+\beta_{r}$ is negative and therefore not a perfect square, for $n_{K+1}$ large enough. Let $\alpha:=\left(\alpha_{1}, \ldots, \alpha_{s}\right) \in(\mathbb{N} \backslash\{0\})^{s}$ and $\beta:=\left(\beta_{1}, \ldots, \beta_{s}\right) \in \mathbb{Z}^{s}$. We denote by $S_{\alpha \beta}:=\left\{\left(\alpha_{r}, \beta_{r}\right)\right\}_{r=1}^{s}$ the set of all couples $\left(\alpha_{r}, \beta_{r}\right)$, for a given choice of $\alpha \in(\mathbb{N} \backslash\{0\})^{s}$ and $\beta \in \mathbb{Z}^{s}$.

Definition 2.5. We say that a positive integer $n \in \mathbb{N}$ satisfies the "no-square condition" with respect to $S_{\alpha \beta}\left(\mathrm{NSC}-S_{\alpha \beta}\right)$ if for all $r=1 \ldots s, \alpha_{r} n+\beta_{r}$ is not a perfect square.

Fix $\alpha \in(\mathbb{N} \backslash\{0\})^{s}, \beta \in \mathbb{Z}^{s}$. Let $F_{N}$ be the number of positive integers $1 \leq n \leq N$ which satisfy (NSC- $\left.S_{\alpha \beta}\right)$.

Consider a single couple $\left(\alpha_{r}, \beta_{r}\right)$ : the main result in 2 implies that there exist two universal constants $C_{1}, C_{2}$ (which do not depend on $\alpha_{r}, \beta_{r}$ ), such that the number of positive integers $1 \leq n \leq N$ such that $\alpha_{r} n+\beta_{r}$ is a perfect square is at most $C_{1} N^{3 / 5}(\ln N)^{C_{2}}$. Now, a positive integer $n$ fails to satisfy $\left(\mathrm{NSC}-S_{\alpha \beta}\right)$ if and only if $\alpha_{r} n+\beta_{r}$ is a perfect square for at least one of the $s$ couples $\left(\alpha_{r}, \beta_{r}\right)$. Therefore, we deduce that the number of positive integers $\leq N$ which fail to satisfy $\left(\mathrm{NSC}-S_{\alpha \beta}\right)$ is

$$
N-F_{N} \leq C_{1} s N^{3 / 5}(\ln N)^{C_{2}} .
$$

Hence, we have

$$
N-C_{1} s N^{3 / 5}(\ln N)^{C_{2}} \leq F_{N} \leq N
$$

which implies that $F_{N}$ is asymptotic to $N$ as $N \rightarrow+\infty$. In particular, this implies that there are infinitely many positive integers satisfying (NSC- $S_{\alpha \beta}$ ), which in turn implies that one can choose $n_{K+1}$ arbitrarily large and satisfying (NSC- $\left.S_{\alpha \beta}\right)$. This concludes the proof of Proposition 2.4

\section{The model equation}

Recall that

$$
\mathcal{A}_{k}=\left\{n_{k}-2, n_{k}-1, n_{k}+1, n_{k}+2\right\}, \quad \mathcal{A}=\bigcup_{k=1}^{K} \mathcal{A}_{k},
$$

and the notation (1.4). We assume that $\left(n_{k}\right)_{k \geq 1}$ is a sequence which satisfies Proposition 2.4 
Next, we set $\varepsilon=\nu^{1 / 4}$ and make the change of unknown $v=\varepsilon u$. Therefore $v$ satisfies

$$
\left\{\begin{array}{l}
i \partial_{t} v+\partial_{x}^{2} v=|v|^{4} v, \quad(t, x) \in \mathbb{R} \times \mathbb{S}^{1}, \\
v(0, x)=v_{0}(x)=\varepsilon u_{0}(x)
\end{array}\right.
$$

We expand $v$ and $\bar{v}$ in Fourier modes

$$
v(x)=\sum_{j \in \mathbb{Z}} \xi_{j} e^{i j x}, \quad \bar{v}(x)=\sum_{j \in \mathbb{Z}} \eta_{j} e^{-i j x},
$$

and denote by $H=\int_{\mathbb{S}^{1}}\left|\partial_{x} v\right|^{2}+\frac{1}{3} \int_{\mathbb{S}^{1}}|v|^{6}$ the Hamiltonian of (3.1).

We define

$$
P(\xi, \eta)=\frac{1}{3} \int_{\mathbb{S}^{1}}|v(x)|^{6} \mathrm{~d} x=\frac{1}{3} \sum_{\substack{j, \ell \in \mathbb{Z}^{3} \\ \mathcal{M}(j, \ell)=0}} \xi_{j_{1}} \xi_{j_{2}} \xi_{j_{3}} \eta_{\ell_{1}} \eta_{\ell_{2}} \eta_{\ell_{3}},
$$

where $\mathcal{M}(j, \ell)=j_{1}+j_{2}+\cdots+j_{p}-\ell_{1}-\ell_{2}-\cdots-\ell_{p}$ denotes the momentum of the multi-index $(j, l) \in \mathbb{Z}^{2 p}$ or equivalently the momentum of the monomial $\xi_{j_{1}} \xi_{j_{2}} \cdots \xi_{j_{p}} \eta_{\ell_{1}} \eta_{\ell_{2}} \cdots \eta_{\ell_{p}}$.

In this Fourier setting the equation (3.1) reads as an infinite Hamiltonian system

$$
\left\{\begin{aligned}
i \dot{\xi}_{j} & =j^{2} \xi_{j}+\frac{\partial P}{\partial \eta_{j}} & & j \in \mathbb{Z}, \\
-i \dot{\eta}_{j} & =j^{2} \eta_{j}+\frac{\partial P}{\partial \xi_{j}} & & j \in \mathbb{Z}
\end{aligned}\right.
$$

with the property that the real subspace $\bar{\xi}=\eta$ is preserved by the equations of motion.

According to this symplectic structure, the Poisson bracket between two functions $f$ and $g$ of $(\xi, \eta)$ is defined by

$$
\{f, g\}=-i \sum_{j \in \mathbb{Z}} \frac{\partial f}{\partial \xi_{j}} \frac{\partial g}{\partial \eta_{j}}-\frac{\partial f}{\partial \eta_{j}} \frac{\partial g}{\partial \xi_{j}} .
$$

In particular, if $(\xi(t), \eta(t))$ is a solution of (3.2) and $F$ is some regular Hamiltonian function, we have

$$
\frac{d}{\mathrm{~d} t} F(\xi(t), \eta(t))=\{F, H\}(\xi(t), \eta(t))
$$

where

$$
H=N+P=\sum_{j \in \mathbb{Z}} j^{2} \xi_{j} \eta_{j}+\frac{1}{3} \sum_{\substack{j, \ell \in \mathbb{Z}^{3} \\ \mathcal{M}(j, \ell)=0}} \xi_{j_{1}} \xi_{j_{2}} \xi_{j_{3}} \eta_{\ell_{1}} \eta_{\ell_{2}} \eta_{\ell_{3}}
$$

is the total Hamiltonian of the system. It is convenient to work in the symplectic polar coordinates $\left(\xi_{j}=\sqrt{I_{j}} \mathrm{e}^{i \theta_{j}}, \eta_{j}=\sqrt{I_{j}} \mathrm{e}^{-i \theta_{j}}\right)_{j \in \mathbb{Z}}$. Since we have $\mathrm{d} \xi \wedge \mathrm{d} \eta=$ $i \mathrm{~d} \theta \wedge \mathrm{d} I$, the system (3.1) is equivalent to

$$
\left\{\begin{array}{rrr}
\dot{\theta}_{j}=-\frac{\partial H}{\partial I_{j}} & & j \in \mathbb{Z} \\
\dot{I}_{j}= & \frac{\partial H}{\partial \theta_{j}} & j \in \mathbb{Z}
\end{array}\right.
$$

Finally, we define

$$
J=\sum_{j \in \mathbb{Z}} I_{j}=\sum_{j \in \mathbb{Z}} \xi_{j} \eta_{j}=\|v\|_{L^{2}\left(\mathbb{S}^{1}\right)}^{2},
$$

which is a constant of motion for (3.1) and (3.2). 
We now refer to [5. Section 3], and in particular to Proposition 3.1 and Proposition 3.3. It is proven that, thanks to a Birkhoff normal form procedure, that there exists a symplectic change of coordinates $\tau$ close to the identity so that

$$
\bar{H}:=H \circ \tau=N+Z_{6}+R_{10},
$$

where

- $N$ only depends on the actions $\left(I_{k}\right)_{k \in \mathbb{Z}}$;

- $Z_{6}$ is the homogeneous polynomial of degree 6 ;

$$
Z_{6}=\sum_{\mathcal{R}} \xi_{j_{1}} \xi_{j_{2}} \xi_{j_{3}} \eta_{\ell_{1}} \eta_{\ell_{2}} \eta_{\ell_{3}}
$$

- $R_{10}$ is a remainder of order 10 in $(\xi, \eta)$.

Lemma 2.3 and Proposition 2.4 have important consequences about the set $\mathcal{R}$ and therefore about the polynomial $Z_{6}$. Lemma 2.3 implies that the monomials in $Z_{6}$ whose modes are all contained in $\mathcal{A}$ actually contain modes from only one of the clusters $\mathcal{A}_{k}$. This somehow allows to decouple the dynamics on the different clusters (see equation (3.4). Proposition 2.4 implies that no monomial of $Z_{6}$ contains five modes from $\mathcal{A}$ and one outside the set $\mathcal{A}$ and that if a monomial of $Z_{6}$ contains four modes in $\mathcal{A}$ and two modes outside $\mathcal{A}$, then one of these two modes must appear as one of the $\xi_{j}$ 's and the other must appear as one of the $\eta_{j}$ 's. Such properties are crucial in the perturbation analysis (for more details, see [5. Section 5] and Section 4 of the present paper).

As in [5. Section 4], we introduce the model system by setting the initial data $\xi_{j}^{0}=\eta_{j}^{0}=0$ in (3.3) when $j \neq \mathcal{A}$. This induces here the Hamiltonian

$$
\widehat{H}=6 J^{3}+\sum_{k=1}^{K} \widehat{H}_{k},
$$

where

$$
\widehat{H}_{k}=\sum_{j \in \mathcal{A}_{k}} j^{2} I_{j}-9 J \sum_{j \in \mathcal{A}_{k}} I_{j}^{2}+4 \sum_{j \in \mathcal{A}_{k}} I_{j}^{3}+18 I_{a_{2}^{(k)}}^{1 / 2} I_{b_{2}^{(k)}}^{1 / 2} I_{a_{1}^{(k)}} I_{b_{1}^{(k)}} \cos \left(2 \varphi_{0}^{(k)}\right),
$$

with $\varphi_{0}^{(k)}=\theta_{a_{1}^{(k)}}-\theta_{b_{1}^{(k)}}+\frac{1}{2} \theta_{a_{2}^{(k)}}-\frac{1}{2} \theta_{b_{2}^{(k)}}$.

Since $\widehat{H}$ is almost decoupled, we obtain a completely integrable system.

LEMMA 3.1. The system given by $\widehat{H}$ is completely integrable.

Proof. Since $J$ is a constant of motion, this is a direct consequence of 5 , Lemma 4.1]. Indeed, for all $1 \leq k \leq K, \widehat{H}_{k}$ and

$$
K_{1}^{(k)}=I_{a_{1}^{(k)}}+I_{b_{1}^{(k)}}, \quad K_{2}^{(k)}=I_{a_{2}^{(k)}}+I_{b_{2}^{(k)}} \text { and } K_{1 / 2}^{(k)}=I_{b_{2}^{(k)}}+\frac{1}{2} I_{a_{1}^{(k)}},
$$

are constants of motion in involution. 
As in [5], we use the coordinates $(\varphi, K)$ to describe some particular trajectories of $\widehat{H}$. To begin with, $\widehat{H}_{k}$ can be rewritten

$$
\begin{aligned}
\widehat{H}_{k}=\widehat{H}_{k}\left(\varphi_{0}^{(k)}, K_{0}^{(k)}, K_{1}^{(k)}, K_{2}^{(k)},\right. & \left.K_{1 / 2}^{(k)}\right) \\
& =F_{k}\left(K_{1}^{(k)}, K_{2}^{(k)}, K_{1 / 2}^{(k)}\right)+6\left[\left(3 J-2 K_{1}\right) I_{a_{1}} I_{b_{1}}\right. \\
& \left.+\left(3 J-2 K_{2}\right) I_{a_{2}} I_{b_{2}}+3 I_{a_{2}}^{\frac{1}{2}} I_{b_{2}}^{\frac{1}{2}} I_{a_{1}} I_{b_{1}} \cos \left(2 \varphi_{0}\right)\right],
\end{aligned}
$$

for some polynomial $F_{k}$ 1. Next, we define

$$
\varepsilon_{k}=\varepsilon \mathrm{e}^{-n_{k}},
$$

we fix

$$
K_{1}^{(k)}=\varepsilon_{k}^{2}, \quad K_{2}^{(k)}=\frac{1}{2} \varepsilon_{k}^{2} \quad \text { and } \quad K_{1 / 2}^{(k)}=\frac{1}{2} \varepsilon_{k}^{2},
$$

and denote by $K_{0}^{(k)}=I_{a_{1}^{(k)}}$. With the previous choice,

$$
J=\sum_{k \geq 1}\left(K_{1}^{(k)}+K_{2}^{(k)}\right)=\frac{3}{2} \varepsilon^{2} \sum_{k \geq 1} \mathrm{e}^{-2 n_{k}}=C \varepsilon^{2} .
$$

The following rescaling proves to be useful

$$
\varphi_{0}^{(k)}(t)=\varphi^{(k)}\left(\varepsilon_{k}^{4} t\right), \quad K_{0}^{(k)}(t)=\varepsilon_{k}^{2} K^{(k)}\left(\varepsilon_{k}^{4} t\right) .
$$

Define

$$
H_{\star}^{(k)}=\frac{9}{4} K^{(k)}\left(1-K^{(k)}\right)\left[\left(10 J \varepsilon_{k}^{-2}-6\right)+4\left(K^{(k)}\right)^{\frac{1}{2}}\left(1-K^{(k)}\right)^{\frac{1}{2}} \cos \left(2 \varphi^{(k)}\right)\right],
$$

and

$$
H_{\star}=\sum_{k=1}^{K} H_{\star}^{(k)},
$$

then for all $1 \leq k \leq K$, the evolution of $\left(\varphi^{(k)}, K^{(k)}\right)$ is given by

$$
\left\{\begin{aligned}
\dot{\varphi}^{(k)}=-\frac{\partial H_{\star}}{\partial K^{(k)}} & =-\frac{27}{4}\left(1-2 K^{(k)}\right)\left[\left(\frac{10}{3} J \varepsilon_{k}^{-2}-2\right)\right. \\
& \left.+2\left(K^{(k)}\right)^{\frac{1}{2}}\left(1-K^{(k)}\right)^{\frac{1}{2}} \cos \left(2 \varphi^{(k)}\right)\right] \\
\dot{K}^{(k)}=\frac{\partial H_{\star}}{\partial \varphi^{(k)}} & =-18\left(K^{(k)}\right)^{\frac{3}{2}}\left(1-K^{(k)}\right)^{\frac{3}{2}} \sin \left(2 \varphi^{(k)}\right) .
\end{aligned}\right.
$$

Then by [5. Proposition 4.2] we have

Proposition 3.2. For all $k \geq 1$, there exists a $\gamma_{k} \in(0,1 / 2)$ so that if $\gamma_{k}<$ $K^{(k)}(0)<1-\gamma_{k}$ and $\varphi(0)=0$, then there is $T_{k}>0$ so that $\left(\varphi^{(k)}, K^{(k)}\right)$ is a $2 T_{k}$-periodic solution of (3.9) and

$$
K^{(k)}(0)+K^{(k)}\left(T_{k}\right)=1 .
$$

Moreover, $T_{k}:\left(\gamma_{k}, 1 / 2\right) \cup\left(1 / 2,1-\gamma_{k}\right) \longrightarrow \mathbb{R}$ is a continuous function of $K^{(k)}(0)$,

$$
T_{k} \longrightarrow \frac{2 \pi}{9}\left(10 J \varepsilon_{k}^{-2}-3\right)^{-1 / 2} \quad \text { as } \quad K^{(k)}(0) \longrightarrow 1 / 2,
$$

\footnotetext{
${ }^{1}$ The expression of $F_{k}$ which can be found in $\mathbf{5}$ is incomplete and it lacks the dependence of $F_{k}$ on $K_{1 / 2}^{(k)}$. However, this does not affect the results in $\mathbf{5}$.
} 
and

$$
T_{k} \longrightarrow+\infty \quad \text { as } \quad K^{(k)}(0) \longrightarrow \gamma_{k} .
$$

Proof. Denote by $C_{k}=10 J \varepsilon_{k}^{-2}-6$. With (3.6), we have $C_{k} \geq 9$ and $C_{k} \sim$ $c \mathrm{e}^{2 n_{k}}$. Write here $K^{(k)}=K$ and $\varphi^{(k)}=\varphi$. Then, as in the proof of [5] Proposition $4.2]$,

$$
K(1-K)\left(C_{k}+4 K^{\frac{1}{2}}(1-K)^{\frac{1}{2}} \cos (2 \varphi)\right)=\frac{1}{4}\left(C_{k}-2\right)
$$

defines two heteroclinic orbits which link the saddle points $(\varphi, K)=(-\pi / 2,1 / 2)$ and $(\varphi, K)=(-\pi / 2,1 / 2)$. When $\varphi=0$

$$
K^{\frac{1}{2}}(1-K)^{\frac{1}{2}}=D_{k}:=\frac{C_{k}-2}{\sqrt{\left(C_{k}-2\right)^{2}+8\left(C_{k}-2\right)}+C_{k}-2} .
$$

Observe that, since $C_{k} \geq 9>2, D_{k} \in(0,1 / 2)$, and this yields the existence of $\gamma_{k}$.

Moreover, $T_{k}$ is a continuous function of $K^{(k)}(0)$ on $\left(\gamma_{k}, 1 / 2\right) \cup\left(1 / 2,1-\gamma_{k}\right)$ and $T_{k} \longrightarrow+\infty$ when $K^{(k)}(0) \longrightarrow \gamma_{k}$, due to the continuous dependence on initial conditions.

Finally, since the point $(\varphi, K)=(0,1 / 2)$ is a nondegenerate centre, we have that $T_{k}$ approaches the value of the half-period of the linearized system as $K^{(k)}(0) \longrightarrow$ $1 / 2$ (it follows from the Theorem in Chapter 5.C, p.100, 1). Since, in particular, we have

$$
\frac{\partial^{2} H_{\star}}{\partial K^{2}}(0,1 / 2)=-\frac{9}{2}\left(C_{k}+3\right), \quad \frac{\partial^{2} H_{\star}}{\partial \varphi^{2}}(0,1 / 2)=-\frac{9}{2}, \quad \frac{\partial^{2} H_{\star}}{\partial K \partial \varphi}(0,1 / 2)=0,
$$

we deduce

$$
\lim _{K^{(k)}(0) \longrightarrow 1 / 2} T_{k}=\frac{2 \pi}{9 \sqrt{C_{k}+3}}
$$

\section{The perturbation analysis and proof of the main results}

We are now ready to complete the proof of Theorem 1.2 This is a direct application of the results of [5, Section 5].

We now assume that $n_{K} \leq-(\ln \varepsilon) / 8=-(\ln \nu) / 32$, therefore by (3.5) we have for all $k \geq 1$

$$
\varepsilon^{9 / 8} \leq \varepsilon_{k} \leq \varepsilon .
$$

Then we can state the following result, which is analogous to [5. Lemma 5.1].

Lemma 4.1. Assume that there exists $C>0$ so that

$$
\left|\xi_{j}(0)\right|,\left|\eta_{j}(0)\right| \leq C \varepsilon \mathrm{e}^{-n_{k}}, \forall j \in \mathcal{A}_{k}
$$

and

$$
\left|\xi_{p}(0)\right|,\left|\eta_{p}(0)\right| \leq C \varepsilon^{3} \mathrm{e}^{-|p|}, \forall p \notin \mathcal{A} .
$$

Then for all $0 \leq t \leq C \varepsilon^{-6}$,

$$
I_{p}(t)=\mathcal{O}\left(\varepsilon^{6}\right) \text { when } p \notin \mathcal{A},
$$

and

$$
\begin{aligned}
K_{1}^{(k)}(t) & =K_{1}^{(k)}(0)+\mathcal{O}\left(\varepsilon^{10}\right) t \\
K_{2}^{(k)}(t) & =K_{2}^{(k)}(0)+\mathcal{O}\left(\varepsilon^{10}\right) t \\
K_{1 / 2}^{(k)}(t) & =K_{1 / 2}^{(k)}(0)+\mathcal{O}\left(\varepsilon^{10}\right) t .
\end{aligned}
$$


We consider the initial conditions

$$
\begin{aligned}
& K_{1}^{(k)}(0)=\varepsilon_{k}^{2}, \quad K_{2}^{(k)}(0)=\frac{1}{2} \varepsilon_{k}^{2}, \quad K_{1 / 2}^{(k)}(0)=\frac{1}{2} \varepsilon_{k}^{2}, \\
& \text { and }\left|\xi_{j}(0)\right|,\left|\eta_{j}(0)\right| \leq C \varepsilon^{3} \mathrm{e}^{-|j|} \text { for } j \notin \mathcal{A},
\end{aligned}
$$

and for all $1 \leq k \leq K$ we set $\tau_{k}=\varepsilon_{k}^{4} t$. Then thanks to Lemma 4.1 we have

Proposition 4.2. Consider the solution of the Hamiltonian system given by $\bar{H}$ with the initial conditions (4.1). Then for all $1 \leq k \leq K,\left(\varphi^{(k)}, K^{(k)}\right)$ defined by (3.7) satisfies for $0 \leq \tau_{k} \leq \varepsilon^{-6} \varepsilon_{k}^{4}$

$$
\left\{\begin{aligned}
\dot{\varphi}^{(k)}\left(\tau_{k}\right) & =-\frac{\partial H_{\star}}{\partial K^{(k)}}+\mathcal{O}\left(\varepsilon^{2}\right) \\
\dot{K}^{(k)}\left(\tau_{k}\right) & =\frac{\partial H_{\star}}{\partial \varphi^{(k)}}+\mathcal{O}\left(\varepsilon^{2}\right),
\end{aligned}\right.
$$

where $H_{\star}$ is the Hamiltonian (3.8).

4.1. Proof of Theorem 1.2 We choose the initial conditions for $(\varphi, K)$. We take $\varphi^{(k)}(0)=0$ and $\gamma_{k}<K^{(k)}(0)<1-\gamma_{k}$. We also consider the solution $\left(\varphi_{\star}^{(k)}, K_{\star}^{(k)}\right)$ to (3.9) with initial condition $\left(\varphi_{\star}^{(k)}, K_{\star}^{(k)}\right)(0)=\left(\varphi^{(k)}, K^{(k)}\right)(0)$. Then by Proposition 4.2] and [5. Lemma 5.3] [2 for all $0 \leq \tau_{k} \leq \varepsilon^{-5} \varepsilon_{k}^{4}$ we have

$$
\left(\varphi^{(k)}, K^{(k)}\right)\left(\tau_{k}\right)=\left(\varphi_{\star}^{(k)}, K_{\star}^{(k)}\right)\left(\tau_{k}\right)+\mathcal{O}\left(\varepsilon^{2}\right) \tau_{k}+\mathcal{O}\left(\varepsilon^{2}\right) \tau_{k}^{2},
$$

which in turn implies that for all $0 \leq t \leq \varepsilon^{-5}$

$$
\begin{aligned}
K_{0}^{(k)}(t) & =\varepsilon_{k}^{2} K_{\star}^{(k)}\left(\varepsilon_{k}^{4} t\right)+\mathcal{O}\left(\varepsilon^{2} \varepsilon_{k}^{6}\right) t+\mathcal{O}\left(\varepsilon^{2} \varepsilon_{k}^{10}\right) t^{2} \\
\varphi_{0}^{(k)}(t) & =\varphi_{\star}^{(k)}\left(\varepsilon_{k}^{4} t\right)+\mathcal{O}\left(\varepsilon^{2} \varepsilon_{k}^{4}\right) t+\mathcal{O}\left(\varepsilon^{2} \varepsilon_{k}^{8}\right) t^{2} .
\end{aligned}
$$

For $t \leq \varepsilon^{-9 / 2}=\nu^{-9 / 8}$ we get (1.5).

The period of $K_{0}^{(k)}$ is $2 T_{k} \varepsilon_{k}^{-4}=2 T_{k} \varepsilon^{-4} \mathrm{e}^{4 n_{k}}$, thus one has to ask that

$$
2 T_{k} \mathrm{e}^{4 n_{k}} \leq \varepsilon^{-1 / 2}=\nu^{-1 / 8} \text {. }
$$

In view of Proposition 3.2 we have that, depending on the choice of initial data, $T_{k}$ spans at least the open interval $\left(\frac{2 \pi}{9}\left(10 J \varepsilon_{k}^{-2}-3\right)^{-1 / 2},+\infty\right)$, which contains the interval $(\pi /(9 \sqrt{3}),+\infty)$ for all $k$, so we can choose the initial data in such a way that $T_{k}=1 / 2$ for all $k$. Therefore, since $n_{k} \leq n_{K}$, (4.2) is satisfied provided that $n_{K} \leq-1 / 32 \ln \nu$.

4.2. Proof of Corollary 1.3. For each $k$, the period of $v_{k}$ equals the period of $K_{0}^{(k)}$, whose value is $2 \nu^{-1} T_{k} \mathrm{e}^{4 n_{k}}$. In the proof of Theorem 1.2 we have observed that, given any $\bar{T} \in(\pi /(9 \sqrt{3}),+\infty)$, one can choose the initial data in such a way that $T_{k}=\bar{T}$. This, with $n_{k} \leq n_{K}$, implies that, for any given $\bar{\Lambda} \in\left(\pi \mathrm{e}^{4 n_{K}} /(9 \sqrt{3}),+\infty\right)$, one can choose the initial data so that the period of $v_{k}$ is equal to $2 \bar{\Lambda} / \nu$. Therefore, given any $K \geq 1$ and any $\Lambda_{1}, \Lambda_{2}, \ldots, \Lambda_{K}>0$, there exists $N \in \mathbb{N}$ so that, with a proper choice of the initial data, $v_{k}$ has period $2 N \Lambda_{k} / \nu$, if $\nu$ is small enough. It suffices to choose the smallest $N$ such that $N \Lambda_{k}>\pi \mathrm{e}^{4 n_{K}} /(9 \sqrt{3})$ for all $k$, which is admissible if for the chosen $N$ one has $N \Lambda_{k}<\nu^{-1 / 8}$ for all $k$, which is verified provided that $\nu$ is small enough.

\footnotetext{
${ }^{2}$ As here, in [5] Lemma 5.3] there is actually an additional error term $\mathcal{O}\left(\varepsilon^{2}\right) \tau^{2}$. This restrains the main result in [5] to times $t \leq \varepsilon^{-5}=\nu^{-5 / 4}$.
} 


\section{References}

[1] V. I. Arnold. Mathematical Methods of Classical Mechanics. Springer, Berlin, 1989. Second edition.

[2] E. Bombieri and U. Zannier. A note on squares in arithmetic progressions, II. Rend. Mat. Acc. Lincei (2002), s. 9, v. 13, 69-75.

[3] B. Grébert. Birkhoff normal form and Hamiltonian PDEs, Partial differential equations and applications, Sémin. Congr., vol. 15, Soc. Math. France, Paris 2007, pp. 1-46.

[4] B. Grébert, E. Paturel and L. Thomann. Beating effects in cubic Schrödinger systems and growth of Sobolev norms. arXiv:1208.5680.

[5] B. Grébert and L. Thomann. Resonant dynamics for the quintic non linear Schrödinger equation. Ann. I. H. Poincaré - AN, 29 (2012), no. 3, 455-477.

[6] B. Grébert and C. Villegas-Blas. On the energy exchange between resonant modes in nonlinear Schrödinger equations. Ann. I. H. Poincaré - AN, 28 (2011), no. 1, 127-134.

Laboratoire de Mathématiques J. Leray, Université de Nantes, UMr CNRS 6629, 2, Rue de la Houssinière, 44322 Nantes Cedex 03, France

E-mail address: emanuele.haus@univ-nantes.fr

Laboratoire de Mathématiques J. Leray, Université de Nantes, UMr CNRS 6629, 2, Rue de la Houssinière , 44322 Nantes Cedex 03, France

E-mail address: laurent.thomann@univ-nantes.fr 